\title{
Reductio ad absurdum from a dialogical perspective
}

\author{
Catarina Dutilh Novaes ${ }^{1}$
}

Published online: 8 April 2016

(c) The Author(s) 2016. This article is published with open access at Springerlink.com

\begin{abstract}
It is well known that reductio ad absurdum arguments raise a number of interesting philosophical questions. What does it mean to assert something with the precise goal of then showing it to be false, i.e. because it leads to absurd conclusions? What kind of absurdity do we obtain? Moreover, in the mathematics education literature number of studies have shown that students find it difficult to truly comprehend the idea of reductio proofs, which indicates the cognitive complexity of these constructions. In this paper, I start by discussing four philosophical issues pertaining to reductio arguments. I then briefly present a dialogical conceptualization of deductive arguments, according to which such arguments (especially mathematical proofs) are best understood as a dialogue between two (fictitious) participants-Prover and Skeptic. Finally, I argue that many of the philosophical and cognitive difficulties surrounding reductio arguments are dispelled or at least further clarified once one adopts a dialogical perspective.
\end{abstract}

Keywords Reductio ad absurdum · Mathematical proofs · Dialogues

\section{Introduction}

In his influential commentary on Euclid's Elements, the fifth century Neo-platonic philosopher Proclus defines reductio ad absurdum arguments in the following terms:

Catarina Dutilh Novaes

c.dutilh.novaes@rug.nl

1 Faculty of Philosophy, University of Groningen, Groningen, The Netherlands 
Every reduction to impossibility ${ }^{1}$ takes the contradictory of what it intends to prove and from this as a hypothesis proceeds until it encounters something admitted to be absurd and, by thus destroying its hypothesis, confirms the proposition it set out to establish. (Proclus, commentary on Euclid's Elements, p. 255 [Morrow 1970])

Schematically, such arguments can be represented $\mathrm{as}^{2}$ :

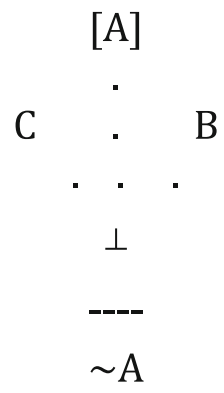

( $\mathrm{B}$ and $\mathrm{C}$ represent additional premises used to derive the absurdity together with the assumption; the set of auxiliary premises is assumed to be consistent.) One noteworthy variation of this schema is when the initial hypothesis is a negative statement of the form 'it is not the case that A'; this will then lead to the conclusion that it is not the case that it is not the case that $\mathrm{A}(\sim \sim \mathrm{A})$, which by the classical rule of double negation elimination is equivalent to $\mathrm{A}$. (Intuitionists do not accept double negation elimination, and thus do not accept reductio arguments of this second kind as establishing A; for them, the argument stops at $\sim \sim \mathrm{A}$.) Notice that the impossibility reached at the penultimate step can be of different kinds: it can be an outright contradiction, but it can also be just some "preposterous implication" (Dennett 2014, 29) of the initial hypothesis. (As we will see, the very status of this absurdity/impossibility raises a number of questions.)

Reductio ad absurdum arguments have many remarkable features. One of them is the productive use made of impossibility/absurdity; rather than representing the end of the road, as it were, in a reductio argument an impossibility allows for the establishment of the truth of a given statement. Reductio arguments reveal the decisive role that the impossible can play in reasoning, and thus once again

\footnotetext{
1 Aristotle already referred to reductio arguments as arguments 'through the impossible', e.g. in the Prior Analytics.

2 Square brackets are used to indicate that the initial proposition is not asserted but rather merely assumed as a hypothesis. However, what it means to make assumptions and thus to produce a proof by hypothesis is still debated (Schroder-Heister 2016). In proof theory, assumptions may be discharged in numerous ways, for example if the assumption of A leads to B, then by stating ' $\mathrm{A} \rightarrow \mathrm{B}$ ' the assumption is discharged. What this means is that, in a reductio proof, once the assumption of $\mathrm{A}$ is shown to lead to absurdity, the statement 'A $\rightarrow \perp$ ' discharges the initial assumption, which is thus no longer 'with us', so to speak, when we then conclude $\sim$ A. (Incidentally, for intuitionists, 'A $\rightarrow \perp$ ' defines the very meaning of $\sim$ A.) We will come back to the roles of assumptions and what it means to discharge them later on.
} 
highlight the need for a satisfactory, fine-grained account of what impossibility is and how to represent it. $^{3}$

Indeed, these arguments are widely used in a number of fields of inquiry, in particular in philosophy and in mathematics. It is sometimes claimed (e.g. Dennett $2014,29)$ that reductio arguments are also used by non-specialists in mundane situations, but this is ultimately an empirical question on which I will have more to say later. At any rate, it is indisputable that these arguments figure prominently both in philosophy and in mathematics. According to the mathematician G. H. Hardy:

Reductio ad absurdum, which Euclid loved so much, is one of a mathematician's finest weapons. It is a far finer gambit than any chess gambit: a chess player may offer the sacrifice of a pawn or even a piece, but a mathematician offers the game. (Hardy 1940, p. 94)

Reductio ad absurdum proofs have been in use in mathematics for millennia, as attested by their frequent use in Euclid's Elements (which is a reflection of by-then well established mathematical practices). It is fair to say that mathematics without reductio proofs would simply not be mathematics as we know it.

Similarly in philosophy (and what has broadly counted as philosophy throughout its history, e.g. including so-called 'natural philosophy'-physics), reductio ad absurdum arguments have played a crucial role. Dennett $(2014,29)$ describes it as "the crowbar of rational inquiry, the great lever that enforces consistency". Some of the oldest, most venerable philosophical arguments are reductios or at least have a reductio-like structure, such as Zeno's paradoxes. Indeed (and this is one of the main claims of this paper), dialectical refutations - the kind of thing that Socrates does in Plato's dialogues when he shows that his interlocutor's discursive commitments are collectively incoherent—can be viewed as the genealogical ancestors ${ }^{4}$ of reductio arguments.

However, despite their ubiquity and significance, reductio ad absurdum arguments raise a number of philosophical issues that suggest reductios are not nearly as straightforward as one might think. In effect, it has often been remarked that reductio proofs are on the whole less explanatory than direct proofs, typically showing that something is the case but not why it is the case. ${ }^{5}$ Moreover (and relatedly), despite claims to the effect that "people use it all the time", 6 reductio

\footnotetext{
3 Admittedly, I will not have that much to say on how to solve this specific issue here.

4 The concept of genealogy in use here is the one I developed in Dutilh Novaes (Dutilh Novaes 2015a). The main point to keep in mind for the present purposes is that if $\mathrm{X}$ is a genealogical ancestor of $\mathrm{Y}$, then $\mathrm{Y}$ has retained traces of $\mathrm{X}$, but it has also undergone some significant modifications.

5 In the history of philosophy, a number of authors have raised concerns regarding the cogency and explanatoriness of reductio proofs. In the Port Royal Logic (1662) for example, Arnauld and Nicole say: “"Such Demonstrations [reductios] constrain us indeed to give our Consent, but no way clear our Understandings, which ought to be the principal End of Sciences: for our Understanding is not satisfied if it does not know not only that a thing is, but why it is? which cannot be obtain'd by a Demonstration reducing to Impossibility". [Quoted in (Lange 2014, 491)] More recently, the later Wittgenstein raised a number of concerns regarding reductio proofs (see Ramharter 2010).
}

6 D. Dennett in video available at http://bigthink.com/videos/daniel-dennett-on-reductio-ad-absurdumthe-philosophers-crowbar. 
arguments appear to be cognitively demanding, as attested by the literature in mathematics education on the difficulties emerging when teaching the technique of reductio proofs to students. Indeed, in a reductio argument, we are asked to start by assuming the impossible, and then to proceed towards showing that it is in fact impossible.

Now, given the centrality of this method both in mathematics and in philosophy, it seems that we would do well to investigate the extent to which it is a trustworthy, reliable method of investigation and argumentation, in particular by addressing relevant cognitive as well as philosophical issues. It will become apparent that the legitimacy of reductio arguments depends crucially on a few (somewhat contentious) assumptions.

The framework to be adopted for the present analysis of reductio arguments is a dialogical perspective. It will be argued that the very concept of deductive arguments/proofs is inherently dialogical, and this holds even more clearly of reductio arguments. While a dialogical conception of reductio ad absurdum is not entirely novel, ${ }^{7}$ it deserves to be investigated more systematically, especially if one is to take seriously the idea that dialectical refutations (which are thoroughly dialogical) may be viewed as the genealogical ancestors of reductio arguments. This perspective also highlights the more strongly adversarial nature of reductio arguments when compared to other kinds of arguments, in a sense to be clarified in due course.

The paper proceeds as follows: I start with a discussion of the cognitive as well as philosophical problems pertaining to the notion of reductio ad absurdum, thus spelling out some of the challenges that need to be met so as to ensure the reliability and cogency of reductio arguments. In the second part, the general dialogical conception of deduction is presented, which ensures that the move to a dialogical perspective when discussing reductio arguments specifically is not ad hoc; to the contrary, it is a perfectly coherent move in light of (what I claim is) the inherently dialogical nature of deduction in general. In the third and final part, we return to the difficulties and issues discussed in the first part, now equipped with the dialogical conception of deduction. This perspective will shed new light on these issues, (dis)solving some of them and making others at least more tractable.

\section{Problems with reductio proofs: cognitive and philosophical}

For philosophers and mathematicians having been suitably 'indoctrinated' in the relevant methodologies, the issues pertaining to reductio ad absurdum arguments may not become immediately apparent, given their familiarity with the technique. And so, to get a sense of what is problematic about such arguments, let us start with a somewhat dramatic but in fact quite accurate account of what we could describe as

\footnotetext{
7 "It's the sort of general purpose crowbar of rational argument where you take your opponent's premises and deduce something absurd from them." (Dennett, video; my emphasis).
} 
the 'phenomenology' of producing a reductio argument, in the words of mathematics educator U. Leron:

We begin the proof with a declaration that we are about to enter a false, impossible world, and all our subsequent efforts are directed towards 'destroying' this world, proving it is indeed false and impossible. (Leron 1985, 323)

In other words, we are first expected to postulate this impossible world (which we believe to be impossible, given that our very goal is to refute the initial hypothesis), and then are required to show that this impossible world is indeed impossible. The first step already raises a number of issues (to be discussed shortly), but the tension between the two main steps (postulating a world, as it were, and then proceeding towards destroying it) is perhaps even more striking. As it so happens, these are not the only two issues that arise once one starts paying attention.

To expose the puzzling nature of reductio arguments, let us start with a discussion of why these arguments appear to be cognitively demanding-that is, if we are to believe findings in the mathematics education literature as well as anecdotal evidence (e.g. of those with experience teaching the technique to students).

\subsection{Cognitive aspects}

Whether reductio ad absurdum arguments are cognitively demanding is obviously by and large an empirical question. Going beyond purported anecdotal evidence that "people" seem to be using this argumentative strategy "all the time", the level of difficulty encountered by those learning to follow and produce such arguments should tell us something about the cognitive challenges involved. Indeed, although there have been few systematic studies of how people fare when reasoning by means of reductios, there is a small but interesting literature in mathematics education that is highly relevant for the present investigation. There is general consensus among educators that students receiving mathematical training tend to find proofs in general difficult, and some kinds of proofs specifically, including reductio proofs, specially difficult. ${ }^{8}$ In particular, students often seem to experience a lack of conviction in reductio ad absurdum proofs, even if they can produce them.

These findings seem to be somewhat in tension the claim that "people use [reductios] all the time"; if they do, why is it so hard for students to accept and internalize this argumentative strategy in the context of mathematical instruction? ${ }^{9}$ And what exactly is so hard about reductios? To address these questions, we may want to pay attention to what students themselves say about their experiences with

\footnotetext{
8 "Research into students' ability to follow or produce proofs... confirms that students find proof difficult, with proofs by (mathematical) induction and proofs by contradiction presenting particular difficulties." (Robert and Schwarzenberger 1991, 130).

9 It may be argued that what students find difficult is the regimented mode of presentation of reductios in mathematics, as well as the fact that they are required to reason in a context-free manner, which runs counter spontaneous reasoning practices. (I owe this point to Uri Leron).
} 
such proofs (even if there are limits to how much introspection can tell us about cognitive processes).

In Antonini and Mariotti (2008), two interview protocols are discussed, where two university students report on their experiences and attitudes towards reductio proofs; they tell us a great deal about what seems to be going on when people formulate such proofs. One student, Maria (majoring in pharmaceutical sciences, final year, and having familiarity with mathematical proofs), offers the following remarks while discussing with the interviewer the possibility of formulating a concrete proof by reductio at absurdum (a proof that, if $a b=0$, then either $a=0$ or $b=0)$ :

[...] well, assume that $a b=0$ with $a$ different from 0 and $b$ different from $0 \ldots$ I can divide by $b \ldots a b / b=0 / b \ldots$ that is $a=0$. I do not know whether this is a proof, because there might be many things that I haven't seen.

Moreover, so as $a b=0$ with $a$ different from 0 and $b$ different from 0 , that is against my common beliefs [Italian: "contro le mie normali vedute"'] and I must pretend to be true, I do not know if I can consider that $0 / b=0$. I mean, I do not know what is true and what I pretend is true.

[Interviewer: Let us say that one can use that $0 / b=0$.]

It comes that $a=0$ and consequently ... we are back to reality. Then it is proved because ... also in the absurd world it may come a true thing: thus I cannot stay in the absurd world. The absurd world has its own rules, which are absurd, and if one does not respect them, one comes back.

But my problem is to understand which are the rules in the absurd world, are they the rules of the absurd world or those of the real world? This is the reason why I have problems to know if $0 / b=0$, I do not know whether it is true in the absurd world. [...]

In the case of the zero-product, I cannot pretend that it is true, I cannot tell myself such a lie and believe it too! (Antonini and Mariotti 2008, 406; emphasis added)

Maria raises a number of issues that for her are cognitive/epistemic issues, but which capture much of what also appears to be philosophically suspicious about reductio proofs more generally. For example, she considers the possibility that she may have overlooked other options; as we'll discuss shortly, a reductio argument only works on the assumption that the enumeration of cases is exhaustive and all alternative possibilities have been considered (each leading to absurdity). She seems particularly bothered by the cognitive dissonance of having to assume that which she believes to be false- "I cannot tell myself such a lie and believe it too!" Maria also wonders whether in the absurd world that is postulated at the beginning of the proof (the world where $a b=0$ but $a \neq 0$ and $b \neq 0$ ), the usual rules of the actual world still hold, so that the reasoning can proceed in the usual way. Indeed, once one accepts such a blatant absurdity, what guarantee do we have that other absurdities will not arise? 
Another student, Fabio (majoring in physics, final year), offers equally insightful considerations:

Yes, there are two gaps, an initial gap and a final gap. Neither does the initial gap is comfortable: why do I have to start from something that is not? [...] However, the final gap is the worst, [...] it is a logical gap, an act of faith that I must do, a sacrifice I make. The gaps, the sacrifices, if they are small I can do them, when they all add up they are too big.

My whole argument converges towards the sacrifice of the logical jump of exclusion, absurdity or exclusion... what is not, not the direct thing. (Antonini and Mariotti 2008, 407; emphasis added)

Like Maria, Fabio feels uncomfortable with the idea of starting from something that he believes to be false. But to him, the most disturbing aspect of a reductio proof is the last step, from absurdity to the contradictory of the initial hypothesis; he describes this step as an 'act of faith'. He also notes the cumulative effect of the cognitive 'sacrifices' he has to make; each of them individually is not so bad, he says, but there are just too many of them involved in a reductio proof.

Naturally, these are the testimonies of just two students: for all we know, they are not representative of how reasoners in general view reductio ad absurdum. However, there is rather strong support in the mathematics education literature for the general idea of cognitive difficulties related to reductio proofs, and thus other reasoners may well be facing similar issues. In particular, it is interesting to notice that, even if they master the technique in terms of being able to produce reductio proofs themselves, students may still feel that these proofs are not entirely trustworthy from an epistemic perspective; indeed, they are often left unconvinced (as Maria and Fabio), perhaps because such proofs are not sufficiently explanatory.

Additional empirical investigation of reasoning abilities with reductio ad absurdum is required to further confirm these findings; but for our purposes, what Maria and Fabio tell us about these proofs provides exactly the right starting point to formulate some of the philosophical issues arising in connection with reductio arguments.

We can think of a reductio ad absurdum as having three main components, following Proclus' description cited above:

(i) Assuming the initial hypothesis.

(ii) Leading the hypothesis to absurdity.

(iii) Concluding the contradictory of the initial hypothesis.

In what follows I discuss two problems pertaining to (i) in Sect. 2.2, and two problems pertaining to (iii) in Sect. 2.3. (ii) is not itself unproblematic, and we have seen for example that Maria worries whether the 'usual' rules for reasoning still apply once we've entered the impossible world established by (i). Moreover, the problematic status of (i) arises to a great extent from its perceived pragmatic conflict with (ii). But the focus from now on will be on issues arising in connection with (i) and (iii). 


\subsection{Problems with 'assuming the impossible'}

A reductio proof starts with the assumption of precisely that which we want to prove is impossible (or false). As we've seen, this seems to create a feeling of cognitive dissonance in (some) reasoners: "I do not know what is true and what I pretend [to be] true." (Maria) This may seem surprising at first sight: don't we all regularly reason on the basis of false propositions, such as in counterfactual reasoning? ("If I had eaten a proper meal earlier today, I wouldn't be so damn hungry now!") However, as a matter of fact, there is considerable empirical evidence suggesting that dissociating one's beliefs from reasoning is a demanding task, cognitively speaking (to 'pretend that something is true', in Maria's terms). The belief bias literature, for example, has amply demonstrated the effect of belief on reasoning, even when participants are told to focus on the connections between premises and conclusions exclusively, not on their beliefs about them. Moreover, empirical studies of reasoning behavior among adults with little to no schooling show their reluctance to reason with premises of which they have no knowledge (Harris 2000; Dutilh Novaes 2013). From this perspective, reasoning on the basis of hypotheses or suppositions may well be something that requires some sort of training (e.g. schooling) to be mastered.

For our purposes, it may be useful to distinguish a number of different cases involving reasoning with false or impossible hypotheses:

- The hypothesis may be false or impossible, without me in fact knowing that it is false or impossible (indeed, I may be in the process of investigating precisely that). ${ }^{10}$

- The hypothesis may be false or impossible, and I believe it to be false or impossible, but I want to see what would be the case if it were true (counterfactual reasoning).

- The hypothesis may be false or impossible, I believe it to be false or impossible, and I am assuming it precisely with the goal of showing it to be false or impossible (a classical reductio argument).

In the narrow sense adopted here, only the last case counts as true reductios. My claim is that, while the first and the second cases describe cognitive activities that human beings regularly engage in, outside circles of specialists, the third case introduces a much stronger component of pragmatic awkwardness: to assume something precisely with the goal of showing it to be false. In 's terms (cited above), this corresponds to the act of postulating a false/impossible world only to proceed to 'destroy' it (show it to be false/impossible). Maria describes it as telling herself a 'lie' and having to believe it.

Perhaps this pragmatic awkwardness arises only due to a reasoner's failure to appreciate the difference between a categorical assertion and an assumption/

\footnotetext{
10 See Wittgenstein's distinction between contradictions to start and contradictions to end with, as discussed in Ramharter (2010).
} 
hypothesis. Indeed, in the examples typically offered to illustrate the purported pervasiveness of reductio arguments 'in the wild', one usually encounters arguments formulated as a conditional or hypothetical sentence ("If he gets here in time for supper, he'll have to fly like Superman"). ${ }^{11}$ However, the conventions for the formulation of a reductio proof in mathematics do not display this general structure perspicuously. Usually, such proofs start with 'Suppose that A'; now, while the 'Suppose that...' operator should function in much the same way, judging from Maria's and Fabio's reports it seems that reasoners feel they must truly commit to the truth of the initial hypothesis while leading it towards absurdity. In effect, this is precisely what seems to be so cognitively demanding: the conflicting roles of 'pretending' to believe the hypothesis and of working towards its very destruction.

The distinction between the initial assumption being merely false or else impossible is also worth discussing in some detail. On most theories of (mental) propositional attitudes, having an attitude towards a false proposition is for the most part unproblematic: false propositions are much like true propositions in terms of their semantics (spelled out in e.g. truth-conditional terms, or use-based accounts, or what have you). Just as I can believe something that is false, I should be able to suppose, i.e. entertain the possibility of, something that is false (even if I know it to be false, unlike with belief).

But matters become considerably more delicate once we are dealing with impossible propositions. While it is not necessarily the case that every reductio argument will start by assuming an impossibility, this is indeed what happens in many paradigmatic cases-certainly if we accept that mathematical truths are necessary truths, and thus mathematical falsities are necessary falsities, i.e. impossibilities. In the Tractatus, Wittgenstein (in)famously claimed that impossibility cannot be expressed, as it cannot be depicted; impossible thoughts cannot be conceived, and impossible propositions cannot be meaningfully formulated. Even though most of us have surely outgrown the Tractarian conception of propositions, we still seem to be stuck with the problem of providing a satisfactory philosophical account of impossible thoughts. As well put by Jago:

Impossible thoughts might appear nothing more than a quirky feature of the way we can meaningfully represent the world around us. It may come as something of a surprise, therefore, to learn that just about every major philosophical theory of content and meaning is unable to account for impossible thoughts. Moreover, a wide variety of philosophical views converge in a kind of pressure group against the existence of impossible thoughts. (Jago 2014, 3)

Jago then goes on to detail how and why different philosophical frameworks fail miserably to account for impossible thoughts and impossible content. What is particularly unsatisfying is that these frameworks (including the Tractarian framework) tend to treat all impossible thoughts/propositions as having the same content: in possible world semantics, for example, all impossible propositions have

11 Dennett, video (see footnote 6). 
the same meaning, namely they correspond to the empty set (the domain being the collection of all possible worlds). But the initial assumptions of reductio proofs in mathematics surely do not all have the same content. If they did, each such reductio proof would be viewed as essentially identical to all others, and this is obviously an undesirable result. ${ }^{12}$

Moreover, specific mathematical cases suggest that entertaining the initial (impossible) hypothesis may be a rather difficult (if not impossible) conceptual achievement. For example, according to (the later) Wittgenstein (Ramharter 2010), in Cantor's diagonal argument we are asked to suppose something that we simply cannot comprehend: what does it even mean to make a list of all real numbers between 0 and 1 ? (The proof then goes on to prove that this cannot be done, but even before that it is not clear how to conceptualize the hypothesis.) Similarly, in Euclid's classical proof of the infinity of the primes, we are asked to suppose there is a largest prime number (only to conclude later on that there is no such thing): can we truly conceive of such a number?

In other words, a philosophical account of reductio ad absurdum requires a sufficiently fine-grained account of impossible thoughts/propositions if we are to make sense of the initial step, that of putting forward the initial hypothesis. Naturally, there are many other reasons to look for adequate theories of the impossible, and some proposals have been put forward (e.g. Jago 2014). Here, I will not venture into offering yet another theory of impossible thoughts; for my purposes, it is enough to note that this is yet another point where reductio ad absurdum is not a straightforward affair from a philosophical perspective.

In sum, there are two problems with the initial speech act in a reductio: the general problem of representing impossible content so as to make sense of the speech act of assuming an impossibility; and the linguistic/pragmatic, equally serious problem of the awkwardness of putting forward a proposition (even if as an assumption or hypothesis), 'pretending' to believe it, precisely in order to show it to be false/impossible. It is this second problem which will receive a natural solution once one adopts a dialogical perspective.

\subsection{Problems with 'jumping to conclusions'}

One worry we may have concerning reductio arguments is what could be described as 'the culprit problem'. This is not a worry clearly formulated in the protocols described above, but one which has been raised a number of times when I presented this material to different audiences. ${ }^{13}$ The basic problem is: we start with the initial assumption, which we intend to prove to be false, but along the way we avail ourselves of auxiliary hypotheses/premises. Now, it is the conjunction of all these premises and hypotheses that leads to absurdity, and it is not immediately clear whether we can single out one of them as the culprit to be rejected. For all we know,

\footnotetext{
${ }^{12}$ What's more, on some accounts, there are only two mathematical propositions: the empty set and the set of all possible worlds. That is, all mathematical statements are either contradictions or tautologies.

13 The worry was raised in particular by Mic Detlefsen and Mathieu Marion.
} 
others may be to blame, and so there seems to be some arbitrariness involved in singling out one specific ingredient as responsible for things turning sour.

To be sure, in most practical cases this will not be a real concern; typically, the auxiliary premises we avail ourselves of are statements to which we attribute a higher degree of epistemic confidence (for example, because they have been established by proofs that we recognize as correct). But it remains of philosophical significance that absurdity typically arises from the interaction between numerous elements, any of which can in theory be held to be responsible for the absurdity. A reductio argument, however, relies on the somewhat contentious assumption that we can isolate the culprit.

Still, culprit considerations do not seem to be what motivates Fabio's dramatic description of this last step as "an act of faith that I must do, a sacrifice I make". Why is this step problematic then? Well, in first instance, what is established by leading the initial hypothesis to absurdity is that it is a bad idea to maintain this hypothesis (assuming that it can be reliably singled out as the culprit, e.g. if the auxiliary premises are beyond doubt). How does one go from it being a bad idea to maintain the hypothesis to it being a good idea to maintain its contradictory?

It may be convenient at this point to notice that a reductio ad absurdum has some interesting similarities with proofs by cases. In a typical proof by cases, if one wants to establish that all $x$ have property Y, for example, it may be helpful to divide the class of $x$ things into sets, and then prove for each of the sets that all its members have property $\mathrm{Y}$. More generally, a proof by cases starts with a presumably exhaustive enumeration of cases-say, A, B, C, and D-and then goes on to show that if $\mathrm{A}$, then $\mathrm{Z}$; if $\mathrm{B}$, then $\mathrm{Z}$; if $\mathrm{C}$, then $\mathrm{Z}$; if $\mathrm{D}$, then $\mathrm{Z}$. Now, on the assumption that the enumeration is exhaustive, if follows unconditionally that $Z$, in all circumstances.

A reductio ad absurdum also starts with the tacit assumption of an exhaustive enumeration of cases: for a given proposition $\mathrm{A}$, either $\mathrm{A}$ is the case or its contradictory is the case, and not both (this follows from excluded middle and the principle of non-contradiction). Once it is shown that A leads to absurdity, by elimination one concludes that its contradictory must be the case. But notice that, unlike in a typical proof by cases, one of the options has not been investigated at all; one is not required to investigate the other option, that is to assume the contradictory $\sim \mathrm{A}$ to see what happens (after having assumed $\mathrm{A}$ and led it to absurdity). The presupposition is that, since exactly one of the two propositions must obtain, once one of them has been eliminated, the second becomes established even though it has not itself been investigated (just as in a disjunctive syllogism).

There are at least two worries one may voice concerning the cogency of these assumptions. (1) Is the enumeration really exhaustive? (Maria: "there might be many things that I haven't seen.") If it is not, it would be premature to conclude that, since A cannot be the case, then $\sim$ A must be the case; there may be some other option B which hasn't yet been considered. (2) What if both A and $\sim$ A lead to absurdity? We are left in the dark as to whether this might happen if we do not run a similar procedure for $\sim$ A.

While 2 may seem somewhat far-fetched in most contexts, there are actually a number of philosophical examples of situations of this kind, which are often 
described as 'aporetic'. Some of Plato's dialogues, for example the Parmenides and the Theaetetus, can be described in these terms: a (presumably) exhaustive enumeration of cases is presented (e.g. in the Theaetetus, three different definitions of 'knowledge'), all of them are investigated, and all of them are found to lead to absurdity or incoherence. Another such example are Kant's antinomies.

This is not to say that these issues will affect each and every instance of a reductio argument; we may have rock-solid reasons to believe that the enumeration in question is indeed exhaustive; it may well be the case that A leads to absurdities while we have no reason to think that the same will happen with $\sim \mathrm{A}$, perhaps after having investigated the possibility. But clearly these are strong assumptions which we may have reasons to question, in specific cases at least. Concerns of this nature may well be what leads Fabio to describe the last step as 'an act of faith'.

\subsection{Partial conclusions}

In sum, we have identified four challenges to the epistemic cogency of reductio arguments; this was (partially) established on the basis of cognitive considerations (in particular, the testimonies of Maria and Fabio).

1. Representing the impossible

2. Pragmatic awkwardness of the first speech act

3. The culprit problem

4. The 'act of faith' problem

In Sect. 4 I will argue that 2 is fully resolved once one adopts a dialogical conception of reductio arguments; I will also argue that both 3 and 4 arise from the fact that reductio arguments are expected to do something that their genealogical ancestors, dialectical refutations, were not designed to accomplish, namely to establish the truth or falsity of a specific thesis. As for 1, admittedly the dialogical perspective does not seem to have that much to add towards possible solutions, but this is a problem that goes well beyond issues pertaining to reductio arguments alone; indeed, it is a problem we all struggle with.

\section{A dialogical account of deduction}

In this section, I present a brief sketch of the general dialogical conception of deduction that I endorse. ${ }^{14}$ Its relevance for the present purposes is to show that a dialogical conception of reductio ad absurdum arguments is not in any way ad-hoc; indeed, the claim is that this conception applies to deductive arguments in general, and thus a fortiori to reductio arguments. (But I will argue later that the dialogical component is even more pronounced in reductio arguments than in other deductive arguments.) It is important to bear in mind that the account here pertains to so-called

\footnotetext{
14 Spelling out all the details of the account in full would require significantly more space; the interested reader is invited to consult some of my work elsewhere, e.g. Dutilh Novaes (2013, 2015b).
} 
'informal' deductive arguments (such as proofs presented in mathematical journals, informal philosophical arguments etc.), and is thus not tied to any specific formal system - though the general idea can be, and has been, spelled out in terms of various formal systems such as different dialogical logics.

Let us start with what can be described as functionalist questions pertaining to deductive arguments and deductive proofs. What is the point of deductive proofs? What are they good for? Why do mathematicians bother producing mathematical proofs at all? While these questions are often ignored by mathematicians and philosophers of mathematics, they have been raised and addressed by Hersh (1993), Rav (1999), and Auslander (2008), among a few others. One promising vantage point to address these questions is the historical development of deductive proof in ancient Greek mathematics, ${ }^{15}$ and on this topic the most authoritative study remains (Netz 1999). Netz emphasizes the importance of orality and dialogue for the emergence of classical, 'Euclidean' mathematics in ancient Greece:

Greek mathematics reflects the importance of persuasion. It reflects the role of orality, in the use of formulae, in the structure of proofs... But this orality is regimented into a written form, where vocabulary is limited, presentations follow a relatively rigid pattern... It is at once oral and written... (Netz 1999, 297/8)

Netz's interpretation relies on earlier work by Lloyd (1996), who argues that the social, cultural and political context in ancient Greece, and in particular the role of the practice of debating, was crucial for the emergence of the technique of deductive proofs. So from this perspective, it seems that one of the main functions of deductive proofs (then as well as now) is to produce persuasion, in particular what one could call explanatory persuasion: to show not only that something is the case, but also why it is the case. ${ }^{16}$ On this conception, a deductive proof corresponds to a dialogue between the person wishing to establish the conclusion (given the presumed truth of the premises), and an interlocutor who will not be easily convinced and will bring up objections, counterexamples, and requests for further clarification. A good proof is one that convinces a fair but 'tough' opponent. Now, if this is right, then mathematical proof is an inherently dialogical, multi-agent notion, given that it is essentially a piece of discourse aimed at a putative audience (Ernest 1994).

To be sure, there are different ways in which the claim that deductive proofs are essentially dialogical can be understood. For example, the fact that a proof is only recognized as such by the mathematical community once it has been sufficiently scrutinized by trustworthy experts (Auslander 2008) can also be viewed as a dialogical component, perhaps in a loose sense (the 'dialogue' between the

\footnotetext{
15 When it comes to functionalist questions, it makes sense to inquire into what the first practitioners of a given practice thought they were doing, and why they were doing it, when the practice first came about. But this is not to exclude the possibility of shifts of function along the way (Dutilh Novaes 2015a, b).

16 See Mancosu (2011) for explanation in mathematics. For Hersh (1993), proof is also about convincing and explaining, but on his account these two aspects come apart. According to him, convincing is aimed at one's mathematical peers, while explaining is relevant in particular in the context of teaching.
} 
mathematician who formulates a proof and the mathematical community). But in what follows I present a more precise rational reconstruction of the (quite specialized) dialogues that would correspond to deductive proofs.

On this conception, proofs are semi-adversarial dialogues of a special kind involving two participants: Prover and Skeptic. ${ }^{17}$ Prima facie, the (fictitious) participants have opposite goals, and this is why the adversarial component remains prominent: Prover wants to establish the truth of the conclusion, and Skeptic wants to block the establishment of the conclusion (though not 'at all costs'). ${ }^{18}$ The dialogue starts with Prover asking Skeptic to grant certain premises. Prover then puts forward further statements, which purportedly follow from what has been granted. (Prover may also ask Skeptic to grant additional auxiliary premises along the way.) Ultimately, most of the work is done by Prover, but Skeptic has an important role to play, namely to ensure that the proof is persuasive, perspicuous, and valid. Skeptic's moves are: granting premises so as to get the game going; offering a counterexample when an inferential move by Prover is not really necessarily truth-preserving; asking for clarifications when a particular inferential step by Prover is not sufficiently compelling and perspicuous. These three moves correspond neatly to what are arguably the three main features of a deductive proof, so let us comment on each of them in turn.

\subsection{Accepting premises/assuming hypotheses}

One of the key components of deductive reasoning is the dissociation of validity from truth. To reason deductively, one must be prepared to put aside one's own beliefs regarding the premises to see what follows from them. As we've seen above when discussing Maria's and Fabio's experiences with reductio arguments, this is a rather demanding cognitive task, one which arguably requires at least some amount of training to be mastered. ${ }^{19}$ In a dialogical setting, however, in particular the somewhat contrived form of dialogical interaction that is debating, granting premises 'for the sake of the argument' is an integral part of the practice. To assume a hypothesis is thus something like a provisional endorsement of a claim, perhaps something like 'pretending to believe it' for the sake of the argument (as suggested by Maria) in order to engage with the interlocutor. Importantly, in a dialogical setting a participant can also draw the consequences of her interlocutor's commitments, without having committed to the claim herself. There is also the interesting question of when, and how, an assumption is no longer in place, either because it has been discharged (though what this technical notion from proof theory means in informal contexts is not immediately clear), or because it has been withdrawn.

\footnotetext{
17 This terminology comes from the computer science literature on proofs. The earliest occurrence that I am aware of is in Sørensen and Urzyczyn (2006), who speak of prover-skeptic games. The general interplay between the two roles is at the heart of Lakatos' (1976) method of 'proofs and refutations'.

18 The 'semi' qualification pertains to the equally strong cooperative component in a deductive proof, to be discussed in more detail shortly.

19 Further discussion of this point can be found in Harris (2000), Dutilh Novaes (2013).
} 


\subsection{Adversariality: necessary truth-preservation}

In a deductive argument, only necessarily truth-preserving inferential steps are permitted. A dialogical setting allows for the formulation of a compelling rationale for the requirement of necessary truth-preservation: necessarily truth-preserving steps are indefeasible, i.e. there is no counterexample that Skeptic could offer to defeat them. And if each individual inferential step in a proof is indefeasible in this sense, then the proof as a whole constitutes a winning strategy for Prover: no matter what Skeptic does (within the rules of the game), no matter what external information he brings in, Prover will prevail. The idea that a deductive proof corresponds to a winning strategy is of course one of the cornerstones of gametheoretical, dialogical conceptions of logic and proof such as Hintikka's gametheoretical semantics and Lorenzen's dialogical logic [see Hodges (2013) and Keiff (2009) for overviews]. On the picture presented here, necessary truth-preservation comes out as the distinctively adversarial component of these dialogues, which determines who 'wins' and who 'loses': if every step in the argument is necessarily truth-preserving, then Prover wins; if Skeptic successfully provides a counterexample, then Skeptic wins.

\subsection{Cooperation: perspicuity as didactic feature}

However, the absence of counterexamples to specific inferential steps is not the whole story: each individual step of a proof must also be compelling and persuasive on its own. Indeed, a desideratum for Prover is to break down the argumentation into small inferential steps; a proof where each step is necessarily truth preserving but not sufficiently convincing is not a good proof. Notice that purely adversarial considerations cannot explain this feature: big 'leaps' are strategically advantageous for Prover. Indeed, in the limit case, Prover could for example get Skeptic to grant the basic axioms of number theory, and then go on directly to state Fermat's Last Theorem as the conclusion: this 'inferential step' would be immune to counterexamples (as established by Wiles' proof of FLT), but obviously such a 'proof' would be an utter failure in that it would not achieve the persuasive, explanatory function of a proof. ${ }^{20}$ This is why one of the moves available to Skeptic is to request for further clarification whenever Prover moves too quickly, so to speak.

From this point of view, a deductive proof (or a deductive argument more generally) is characterized by a complex interplay between adversariality and cooperation: the participants have opposite goals and 'compete' with one another at a lower level, but they are also engaged in a common project to establish the truth or falsity of a given conclusion (given the truth of the premises) in a way that is not only persuasive but also (hopefully) elucidatory. (That is, the ideal of explanatoriness is essentially cooperative.) If both participants perform to the best of their

\footnotetext{
${ }^{20}$ What it takes for a proof to be explanatory arguably goes beyond each of its individual steps being convincing; an explanatory proof should also somehow reveal the conceptual connections involved. But of course, there is still much debate on what it takes for a proof to be explanatory (Mancosu 2011).
} 
abilities, then the common goal of producing novel, verified mathematical knowledge will be optimally achieved. ${ }^{21}$

\subsection{The internalization of Skeptic}

At this point, the reader may be wondering: this is all very well, but obviously deductive proofs are not really dialogues! They are typically presented in writing rather than produced orally (though of course they can also be presented orally, for example in the context of teaching), and if at all, there is only one 'voice' we hear, that of Prover. So at best, they must be viewed as monologues. My answer to this objection is that Skeptic may have been 'silenced', but he is still alive and well insofar as the deductive method has internalized the role of Skeptic by making it constitutive of the deductive method as such. Recall that the job of Skeptic is to look for counterexamples and to make sure the argumentation is perspicuous. This in turn corresponds to the requirement that each inferential step in a proof must be necessarily truth preserving (and so immune to counterexamples), and that a proof must have the right level of granularity, i.e. it must be sufficiently detailed for the intended audience, in order to achieve its explanatory purpose.

Indeed, the internalization of Skeptic may be identified in the very historical process detailed in Netz (1999) (and alluded to in the passage quoted above) from proofs orally presented, corresponding to actual dialogues (e.g. Socrates' proof of how to double the size of a square in the Meno) to written proofs, such as the ones found in Euclid's Elements. From this perspective, a mathematical proof both is and is not a dialogue: it is a dialogue in that it retains dialogical components (assuming hypotheses, producing indefeasible but convincing arguments), but it is no longer a dialogue properly speaking insofar as one of the participants has been internalized by the method itself, and thus silenced. As Netz puts it, "it is at once oral and written".

\section{A dialogical account of reductio ad absurdum}

Equipped with the dialogical account of deduction just sketched, we can now return to reductio arguments and spell out in detail what it means to say that they are best understood from a dialogical perspective. This in turn will allow us to return to the issues pertaining to reductio ad absurdum discussed in Sect. 2. But before we do this, a short historical excursion on the notion of dialectical refutations is required, given the claim that dialectical refutations can be viewed as the genealogical ancestors of reductio ad absurdum arguments.

\footnotetext{
${ }^{21}$ Compare to what happens in a court of law in adversarial justice systems: defense and prosecution are defending different viewpoints, and thus in some sense competing with one another, but the ultimate common goal is to achieve justice. The presupposition is that justice will be best served if all parties perform to the best of their abilities.
} 


\subsection{Dialectical refutations}

Those familiar with Plato's Socratic dialogues will undoubtedly recall the numerous instances in which Socrates, by means of questions, elicits a number of discursive commitments from his interlocutors, only to go on to show that, taken collectively, these commitments are incoherent. This is the procedure known as an elenchus, or a dialectical refutation. ${ }^{22}$

The ultimate purpose of such a refutation may range from ridiculing the opponent to nobler didactic goals. The etymology of elenchus is related to shame, and indeed at least in some cases it seems that Socrates is out to shame the interlocutor by exposing the incoherence of their beliefs taken collectively (for example, so as to exhort them to positive action, as argued in Brickhouse and Smith (1991)). However, as noted by Socrates himself in the Gorgias (470c7-10), refuting is also what friends do to each other, a process whereby someone rids a friend of nonsense. An elenchus can also have pedagogical purposes, in interactions between masters and pupils. ${ }^{23}$

There has been much discussion in the secondary literature on what exactly an elenchus is, as well as on whether there is a sufficiently coherent core of properties for what counts as an elenchus, beyond a motley of vaguely related argumentative strategies even as documented in Plato's dialogues (Carpenter and Polansky 2002). (A useful recent overview is Wolfsdorf (2013); see also Scott (2002)). For our purposes, it will be useful to take as our starting point the description of the 'Socratic method' in an influential article by Vlastos (1983) [a shorter version of the same argument is to be found in Vlastos (1982)]. In line with earlier work by Richard Robinson, Vlastos distinguishes two kinds of elenchi, the indirect elenchus and the standard elenchus:

Here [in the indirect elenchus] Socrates is uncommitted to the truth of the premise-set from which he deduces the negation of the refutand. This mode of argument is a potent instrument for exposing inconsistency within the interlocutor's beliefs. But it cannot be expected to establish the truth or falsehood of any particular thesis. For this Socrates must turn to standard elenchus. (Vlastos 1982, 711)

He then goes on to describe the alternative, standard elenchus, in the following way:

I argue that this is a search for moral truth ${ }^{24}$ through two-part question-andanswer adversative argument, which normally proceeds as follows:

\footnotetext{
22 However, we should not take Plato's account as telling the whole story about what elenchi were for his immediate predecessors and his contemporaries (Lesher 2002). More likely, the term was used to cover a related but nevertheless diverse collection of argumentative strategies; Plato did not owe the copyright, so to speak. In other words, there may well have been different kinds of elenchi (Castelnerac and Marion 2009; Carpenter and Polansky 2002).

23 For (more recent) instantiations of elenchus-like modes of teaching in the context of mathematics, see Lakatos (1976) and Leron and Ejersbo (2015).

24 Notice that Vlastos restricts the scope of action of elenchi to moral truths, which is an important element of his account, but which will not play any significant role for the present purposes.
} 
1. The interlocutor, "saying what he believes", asserts $p$, which Socrates considers false, and targets for refutation.

2. Socrates obtains agreement to further premises, say $q$ and $r$, which are logically independent of $p$. The agreement is ad hoc: Socrates does not argue for $q$ or for $r$.

3. Socrates argues, and the interlocutor agrees, that $q$ and $r$ entail not- $p$.

4. Thereupon Socrates claims that $p$ has been proved false, not- $p$ true. (Vlastos 1982, 712)

This scheme can be viewed as a dialogical variant of traditional (monological) reductio ad absurdum, where one and the same agent makes the initial hypothesis, goes on to show that it leads to absurdity, and then concludes the contradictory of the initial hypothesis. In the dialogical case, by contrast, we have what could be described as a division of labor between the different participants: the interlocutor claims $p$, and then it is Socrates who shows that commitment to $p$ and to other premises $q$ and $r$ is incoherent, given that $q$ and $r$ entail not- $p$. (Notice also that commitment to $q$ and $r$ is elicited by Socrates in Vlastos' description.) It is then Socrates again who concludes not- $p$, and thus not the interlocutor who had committed to $p$ in the first place.

Vlastos' account of the 'standard elenchus' has been criticized by a number of scholars (e.g. Benson 1987, 1995), who point out that the Socratic elenchus can only have a negative function, that is, to show an interlocutor not that $p$ is false, but merely that $p$ is inconsistent with their other beliefs. In other words, it is step 4 in Vlastos' reconstruction that is particularly contentious, as up to step 3 what has been achieved is merely to show the incoherence of the interlocutor's simultaneous commitment to $p, q$, and $r$. (Step 4 is precisely what Fabio describes as 'an act of faith'.) At this point, the consensus in the literature is essentially that Vlastos' account is incorrect as an account of Socratic elenchus, as found in Plato's dialogues. But one compelling feature of Vlastos' account is his emphasis on the adversarial ('adversative' is the term he uses above) component of a dialectical refutation, even if adversariality is combined with cooperative elements (e.g. the reference to friendship in the Gorgias).

On Vlastos' account, an elenchus comes out as virtually equivalent to a reductio ad absurdum, given that the culmination is the establishment of the truth of a given thesis, namely the contradictory of the interlocutor's initial assertion, through an intermediate stage of incoherence/absurdity (simultaneous commitment to $p, q$, and $r$ ). And if Vlastos' account of elenchus were accurate, then the dialogical nature of reductio ad absurdum would emerge in a rather straightforward way: reductiones ad absurdum and dialectical refutations are essentially the same thing; dialectical refutations are essentially dialogical; thus, reductiones ad absurdum are essentially dialogical.

But of course, it is now clear that Vlastos' account of elenchus is not correct; numerous scholars have argued that his attribution of a positive function to refutations is textually unfounded. However, I still want to claim that dialectical refutations can be viewed as the genealogical ancestors of reductio ad absurdum arguments. The relations of historical priority between logic (philosophy) and 
mathematics in ancient Greece are contentious (Mueller 1974), and given the scarce availability of textual material we may never come to know for sure how exactly these developments took place. But it is reasonable to assume that there was extensive contact between philosophers (and more generally those engaging in the practice of dialectic) and mathematicians (sometimes they were the same people). Moreover, Lloyd (1996) and Netz (1999) have persuasively established the role of orality and debating for the emergence of classical, Euclidean mathematics. Thus, it is not completely unreasonable to suppose that the practice of dialectical refutation may have had some influence in the development of the technique of reductio arguments, which then became pervasive in Greek mathematics. ${ }^{25}$

A case in point is the ambiguous status of Zeno's paradoxes. Indeed, they can be interpreted either as straightforward reductios, or as dialectical refutations. On the first interpretation, the result achieved is the establishment of the truth of Parmenides' theses to the effect that there is no plurality, there is no change, no movement etc., once Zeno shows that assuming that there are such things leads to incoherence. On the second interpretation, by contrast (and this is the position that Plato attributes to Zeno in Parmenides 128a-e), what is achieved is merely to show that the positions of the opponents of Parmenides can lead to absurdity, and thus that they are not obviously correct despite the apparent strangeness of Parmenides' views. On this interpretation, an elenchus would function above all as a 'dialectical silencer' (Castagnoli 2010), whereby the incoherence of the position of one of the interlocutors becomes manifest.

In short, there is a fundamental difference between a reductio argument and a dialectical refutation, namely that the former aims at establishing the truth (or falsity) of a given thesis, whereas the latter can only show that a certain number of claims, when taken collectively, lead to incoherence, without thereby singling any one of them out as false. ${ }^{26}$ However, it still seems apposite to view dialectical refutations as the genealogical ancestors of reductios. And if this is so, then reductio arguments would have clear dialogical origins, and arguably would have retained at least some dialogical aspects.

\subsection{Issues reconsidered}

A general dialogical schema for reductio ad absurdum, following Proclus' description $^{27}$ and inspired by the Socratic elenchus, might look like this:

\footnotetext{
25 Naturally, much more work would be required in order to substantiate the claim that dialectical refutations can be viewed as the genealogical ancestors of reductio arguments, in particular extensive textual analysis. At this point, I offer it as no more than a plausible hypothesis requiring further investigation.

26 One way to outline the similarity between the two might be to view a dialectical refutation as establishing the falsity (indeed, absurdity) of the conjunction of the interlocutor's commitments ( $p, q$ and $r)$.

27 (i) Assuming the initial hypothesis; (ii) leading the hypothesis to absurdity; (iii) concluding the contradictory of the initial hypothesis.
} 
(i) Interlocutor 1 commits to A (either prompted by a question from interlocutor 2, or spontaneously), which corresponds to assuming the initial hypothesis.

(ii) Interlocutor 2 leads the initial hypothesis to absurdity, typically by relying on additional discursive commitments of 1 (which may be elicited by 2 through questions).

(iii) Interlocutor 2 concludes $\sim \mathrm{A}$; interlocutor 1 is then well advised to withdraw her original commitment to A (or one of her other commitments).

The main difference between the monological and the dialogical versions of a reductio is thus that in the latter there is a kind of division of labor that is absent from the former (as noted above). The agent making the initial assumption is not the same agent who will lead it to absurdity, and then conclude its contradictory. And so, the perceived pragmatic awkwardness of making an assumption precisely with the goal of 'destroying' it seems to vanish. Moreover, the adversarial component provides a compelling rationale for the general idea of 'destroying' the initial hypothesis; indeed, while the adversarial component is present in all deductive arguments (in particular given the requirement of necessary truth preservation, as argued above), it is even more pronounced in the case of reductio arguments, that is the procedure whereby someone's discursive commitments are shown to be collectively incoherent since they lead to absurdity. There remains the question of why interlocutor 1 would want to engage in the dialogue at all, but presumably she simply wishes to voice a discursive commitment to A. From there on, the wheel begins to spin, mostly through 2's actions.

Thus, the issue of the pragmatic awkwardness of the first speech act is fully (dis)solved once one adopts the multi-agent, dialogical perspective which ensures different rationales/motivations for the different steps in the argument, carried out by different agents. In the mono-agent case, as a result of the process of internalization of one of the participants described in Sect. 3.4, one and the same agent has to play conflicting roles, which for some reasoners (Maria and Fabio, for example) seems to create a situation of cognitive dissonance.

What about the three other issues discussed in Sect. 2? As already mentioned, the dialogical perspective does not seem to offer any new resources to tackle the issue of how to represent the impossible; we are still saddled with this problem just as we were in the monological case. But the dialogical perspective does have something to offer with respect to the other two issues, namely the culprit problem and the 'act of faith' problem, even if it does not lead to fully-fledged (dis)solutions as in the case of the first speech act problem.

Indeed, the key point is again the idea that dialectical refutations can be viewed as the genealogical ancestors of reductio ad absurdum arguments. Both the culprit problem and the 'act of faith' problem pertain to the last step in a reductio argument, namely the step from absurdity to the final conclusion (which is the contradictory of the initial assumption), and thus to the idea that a positive outcome can be reached - the establishment of the truth (or falsity) of a given claim. As we've seen, most scholars believe (contra Vlastos) that this last step is absent in dialectical refutations, which can only have the negative outcome of establishing the collective incoherence of a group of beliefs/commitments. Indeed, a reductio argument is 
much like a dialectical refutation, except for the last step in a reductio. And thus, we could say that, with the addition of the last step, there is a shift of function from the original practice (refutations) to the new one (reductio arguments), but the shift presupposes resources that the original practice lacks. ${ }^{28}$ In other words, we could say that reductio arguments overstretch the resources contained in the original matrix, and this gives rise to these philosophical difficulties.

This does not mean that reductio arguments, and in particular the last step, are never justified. The point is rather that the last step strongly relies on a number of assumptions, and if these are not in place then the argument does not go through. Regarding the culprit problem, what is required is that all auxiliary assumptions/ premises used in the argument have a higher degree of certainty to us than the initial assumption that is singled out to be rejected. Regarding the act of faith problem, if we can be sure that the enumeration of cases is truly exhaustive (i.e. excluded middle holds in the relevant situation), and that we will not end up in a situation of aporia where all options lead to absurdity, then we can safely conclude not- $p$ after showing that $p$ leads to absurdity. The dialogical perspective (in particular the comparison with dialectical refutations) allows for the identification of these key assumptions, and this in itself represents a solution of sorts to the issues pertaining to the final step in a reductio argument.

Moreover, the fact that in reductio arguments the adversarial component is more pronounced than in direct arguments also offers a partial account of why reductio proofs are typically viewed as less explanatory. Explanatoriness is a cooperative component, which becomes less pronounced when adversariality becomes more pronounced, namely with the goal of showing the global incoherence of an interlocutor's commitments.

\section{Conclusions}

This paper started with a discussion of a number of issues arising in connection with reductio arguments. A reductio ad absurdum may well be a fine weapon, as described by Hardy, but it is one that brings along a number of challenges. We've seen that the mathematics education literature seems to suggest that students tend to find reductio proofs somewhat mystifying, and many of them seem to view such proofs as unpersuasive. In Sect. 2, a brief discussion of two experimental protocols set the stage for the formulation of four philosophical issues arising in connection with reductio arguments: how to represent the impossible; the first speech-act problem; the culprit problem; the 'act of faith' problem.

In Sect. 3 I presented a brief account of a general dialogical conception of deductive arguments, which is largely inspired by the historical development of logic and mathematics in ancient Greece. Equipped with this conception, I then

\footnotetext{
${ }^{28}$ The idea of shifts of function/meaning in genealogical processes is one of the cornerstones of Nietzsche's conception of genealogy, as masterfully discussed in Geuss (1994). I borrowed this idea to articulate the methodology of conceptual genealogy in Dutilh Novaes (2015a), and here it comes to play an important role in explaining what is going on with the culprit problem and the act of faith problem.
} 
returned specifically to reductio arguments in Sect. 4, firstly by offering a brief discussion of dialectical refutations, and secondly by reassessing the issues discussed in Sect. 2 now from a dialogical perspective. The conclusion was that, of the four issues discussed, the dialogical conception can fully solve one of them, the so-called first speech-act problem; it can further shed new light on two of them, namely the culprit problem and the 'act of faith' problem, giving us further clues as to what is problematic about them and helping us isolate the assumptions that need to be in place for the last step in a reductio to be compelling. As for the remaining problem, how to represent the impossible, at this point it is not clear to me how a dialogical perspective could contribute towards the formulation of a theory of the impossible and of representing impossibility, both conceptually and linguistically. But perhaps further reflection will show that it can.

Be that as it may, I submit that the dialogical perspective brings us closer to a better understanding of reductio ad absurdum arguments. While I've adopted a somewhat critical stance at times, the present analysis is not intended as revisionary of current practices, i.e. as the plea for a general ban on the use of such arguments. Rather, the point is to outline the assumptions underlying this argumentative strategy by highlighting its dialogical aspects, and thus hopefully to produce a better understanding of its reach and limits.

Perhaps a potential contribution of the present analysis is to the issue of how to teach the technique of reductio arguments, in mathematics as well as elsewhere, in more effective ways. The traditional mode of presentation of reductio proofs, where the theorem to be proved is stated at the very beginning, followed by 'suppose not...', seems to cause the kind of cognitive dissonance described by Maria and Fabio, especially if the hypothetical status of the initial assumption is not sufficiently highlighted. Instead, if the gist of a reductio proof is presented in dialogical terms, i.e. the goal being to disprove a commitment undertaken by one's opponent, then students may well acquire a better grasp of how to produce such arguments and how to interpret them. Ultimately, this is a hypothesis to be tested empirically. But should it prove to be didactically effective, the approach may well make a difference to how students learn the technique of reductio arguments.

However, while cognitive and pedagogical elements occupy an important place in the present investigation, it remains ultimately philosophical in nature. The goal was to articulate a philosophical account of the nature of reductio ad absurdum arguments, and the main claim is that this is achieved by adopting a dialogical perspective on deductive arguments in general, and on reductio ad absurdum in particular.

Acknowledgments I had the chance to present this material in Groningen, Paris, Nancy, Leeds, Montreal, Munich, and Bristol; thanks to the audiences for insightful feedback. I've also received useful feedback from readers of my blog posts with different instantiations of these ideas. Special thanks go to Uri Leron, Leon Geerdink, Rohan French, and Matthew Duncombe for detailed comments on earlier drafts.

Open Access This article is distributed under the terms of the Creative Commons Attribution 4.0 International License (http://creativecommons.org/licenses/by/4.0/), which permits unrestricted use, distribution, and reproduction in any medium, provided you give appropriate credit to the original 
author(s) and the source, provide a link to the Creative Commons license, and indicate if changes were made.

\section{References}

Antonini, S., \& Mariotti, M. A. (2008). Indirect proof: What is specific to this way of proving? ZDMThe International Journal on Mathematics Education, 40, 401-412.

Auslander, J. (2008). On the roles of proof in mathematics. In B. Gold \& R. Simons (Eds.), Proof and other dilemmas: Mathematics and philosophy (pp. 61-77). Washington, DC: Mathematical Association of America.

Benson, H. H. (1987). The problem of the elenchus reconsidered. Ancient Philosophy, 7, 67-85.

Benson, H. H. (1995). The dissolution of the problem of the elenchus. Oxford Studies in Ancient Philosophy, 13, 45-112.

Brickhouse, T. C., \& Smith, N. D. (1991). Socrates' elenctic mission. Oxford Studies in Ancient Philosophy, 9, 131-160.

Carpenter, M., \& Polansky, R. M. (2002). Variety of Socratic elenchi. In Scott (pp. 89-100).

Castagnoli, L. (2010). Ancient self-refutation: The logic and history of the self-refutation argument from Democritus to Augustine. Cambridge: Cambridge University Press.

Castelnerac, B., \& Marion, M. (2009). Arguing for inconsistency: Dialectical games in the academy. In G. Primiero \& S. Rahman (Eds.), Acts of knowledge: History, philosophy and logic. London: College Publications.

Dennett, D. (2014). Intuition pumps and other tools for thinking. New York: W.W. Norton Company.

Dutilh Novaes, C. (2013). A dialogical account of deductive reasoning as a case study for how culture shapes cognition. Journal of Cognition and Culture, 13, 453-476.

Dutilh Novaes, C. (2015a). Conceptual genealogy for analytic philosophy. In J. Bell, A. Cutrofello, \& P. M. Livingston (Eds.), Beyond the analytic-continental divide: Pluralist philosophy in the twenty-first century (pp. 75-110). New York: Routledge.

Dutilh Novaes, C. (2015b). A dialogical, multi-agent account of the normativity of logic. Dialectica, 69, 587-609.

Ernest, P. (1994). The dialogical nature of mathematics. In P. Ernest (Ed.), Mathematics, education and philosophy: An international perspective (pp. 33-48). London: The Falmer Press.

Geuss, R. (1994). Nietzsche and genealogy. European Journal of Philosophy, 2(3), 274-292.

Hardy, G. H. (1940). A mathematician's apology. Cambridge: CUP.

Harris, P. (2000). The work of the imagination. New York: Wiley/Blackwell.

Hersh, R. (1993). Proving is convincing and explaining. Educational Studies in Mathematics, 24(4), 389-399.

Hodges, W. (2013). Logic and games, In E. N. Zalta (Ed.), The Stanford encyclopedia of philosophy (Spring 2013 ed.). http://plato.stanford.edu/archives/spr2013/entries/logic-games.

Jago, M. (2014). The impossible: An essay on hyperintensionality. Oxford: Oxford University Press.

Keiff, L. (2009). Dialogical logic. In E. N. Zalta (Ed.), The Stanford encyclopedia of philosophy (Summer 2011 ed.). http://plato.stanford.edu/archives/sum2009/entries/logic-dialogical.

Lakatos, I. (1976). Proofs and refutations. Cambridge: CUP.

Lange, M. (2014). Aspects of mathematical explanation: Symmetry, unity, and salience. Philosophical Review, 123(4), 485-531.

Leron, U. (1985). A direct approach to indirect proofs. Educational Studies in Mathematics, 16(3), $321-325$.

Leron, U., \& Ejersbo, L. R. (2015). What is the opposite of cat? A gentle introduction to group theory. International Journal of Mathematical Education in Science and Technology, 47(1), 120-132.

Lesher, J. H. (2002). Parmenidean elenchos. In G. A. Scott (Ed.), Does socrates have a method? rethinking the Elenchus in Plato's dialogues and beyond (pp. 19-35). Pennsylvania State University Press.

Lloyd, G. E. R. (1996). Science in antiquity: The Greek and Chinese cases and their relevance to the problem of culture and cognition'. In D. Olson \& N. Torrance (Eds.), Modes of thought: explorations in culture and cognition (pp. 15-33). Cambridge: CUP. 
Mancosu, P. (2011). Explanation in mathematics. In E. N. Zalta (Ed.), The Stanford encyclopedia of philosophy (Summer 2015 ed.). http://plato.stanford.edu/archives/sum2011/entries/mathematicsexplanation/.

Morrow, G. R. (1970). (tr.) Proclus, a commentary on the first book of Euclid's elements. Princeton: Princeton University Press.

Mueller, I. (1974). Greek mathematics and Greek logic. In J. Corcoran (Ed.), Ancient logic and its modern interpretations (pp. 35-70). Dordrecht: Reidel.

Netz, R. (1999). The shaping of deduction in Greek mathematics: A study in cognitive history. Cambridge: CUP.

Ramharter, E. (2010). Are all contradictions equal? Wittgenstein on confusion in mathematics. In B. Löwe \& T. Müller (Eds.), Philosophy of mathematics: Sociological aspects and mathematical practice (pp. 293-306). London: College Publications.

Rav, Y. (1999). Why do we prove theorems? Philosophia Mathematica, 7(1), 5-41.

Robert, A., \& Schwarzenberger, R. (1991). Research in teaching and learning mathematics at an advanced level. In D. Tall (Ed.), Advanced mathematical thinking (pp. 127-139). Dordrecht: Kluwer.

Schroder-Heister, P. (2016). Open problems in proof-theoretic semantics. In T. Piecha \& P. SchroederHeister (Eds.), Advances in proof-theoretic semantics, trends in logic. Berlin: Springer.

Scott, G. A. (Ed.). (2002). Does Socrates have a method?. University Park: Pennsylvania State University Press.

Sørensen, M. H., \& Urzyczyn, P. (2006). Lectures on the Curry-Howard isomorphism, volume 149 (studies in logic and the foundations of mathematics). New York: Elsevier.

Vlastos, G. (1982). The socratic elenchus. The Journal of Philosophy, 79(11), 711-714.

Vlastos, G. (1983). The socratic elenchus. Oxford Studies in Ancient Philosophy, 1, 27-58.

Wolfsdorf, D. (2013). Socratic philosophizing. In J. Bussanich, \& N. D. Smith (Eds.), The Bloomsbury companion to Socrates. Continuum. 